\title{
MS03-P08 | The Role Of Odd In The Biosynthesis Of Medically Important
}

\section{AlkALOIDS}

Kot, Ewa (Jerzy Haber Institute of Catalysis and Surface Chemistry Polish Academy of Sciences, Krakow, POL); Kurpiewska, Katarzyna (Jerzy Haber Institute of Catalysis and Surface Chemistry Polish Academy of Science, Krakow, POL); Borowski, Tomasz (Jerzy Haber Institute of Catalysis and Surface Chemistry Polish Academy of Science, Krakow, POL)

Most of drugs belong to substances produced by plants and called with a common name as alkaloids. It is a group of organic compounds with one or more nitrogen atoms which are mostly alkaline. To one of the most popular alkaloids morphine or codeine can be counted; both have similar chemical properties. Morphine is mainly used as a painkiller in heroin addiction or as a counter the effects of opioid overdose. Codeine also can be analgesic but causes the disappearance of feeling hungry [1]. Both, morphine and codeine, belong to alkaloids which are produced in opium poppy (Papaver somniferum) at the morphine biosynthesis pathway [2]. The biosynthesis pathways for morphine and berberine engage 2-oxoglutarate dependent dioxygenases (ODD) [3].

Presented project concerns production, crystallization, catalytic characterization and engineering of plant enzymes belonging to the superfamily of 2-oxoglutarate dependent dioxygenases. Three enzymes: codeine O-demethylase (CODM), thebaine 6-O-demethylase (T6ODM) and protopine O-dealkylase (PODA) are under investigation. Two of them, CODM and T6ODM, catalyze penultimate and final steps in morphine biosynthesis, while PODA can catalyze the 0,0 -demethylenation of methylenedioxy bridges on protopine and on protoberberine alkaloids. All three enzymes can catalyze reactions for a relatively wide range of alkaloids as a substrate [2].

Number of expression and purification experiments were performed so far, including optimization of different bacterial hosts systems, culture conditions and purification protocols. As a result, active and stable enzymes were obtained. Results of biochemical experiments will be presented.

[1] Rang et al., 1995

[2] Runguphan et al., 2012

[3] Farrow and Facchini, 2013 